\title{
Tecnologias de Informação Móveis, Sem Fio e Ubíquas: Definições, Estado-da-Arte e Oportunidades de Pesquisa
}

\author{
Amarolinda Zanela Saccol \\ Nicolau Reinhard
}

\section{RESUMO}

As Tecnologias de Informação Móveis, Sem Fio e Ubíquas figuram entre os principais temas atualmente discutidos na área de Sistemas de Informação, tanto no meio empresarial quanto no meio acadêmico. A crescente aplicação dessas tecnologias faz emergir uma série de questões relativas à sua criação, escolha, adaptação e consequiências de utilização. Contudo, por serem tecnologias relativamente recentes, há certa confusão conceitual em torno de termos como: Tecnologias Móveis (Mobile), Tecnologias Sem Fio (wireless), Tecnologias Ubíquas, Comércio Móvel (m-commerce) e Negócios Móveis ( $m$-business). Esses termos são utilizados em profusão, tanto no mercado quanto na pesquisa acadêmica, muitas vezes sem definição clara do seu significado. Diante desse contexto, este artigo realiza um mapeamento do estado-da-arte da pesquisa sobre as Tecnologias de Informação Móveis, Sem Fio e Ubíquas, apresenta as definições dos principais conceitos relacionados a essas tecnologias, bem como identifica lacunas de conhecimento existentes, indicando oportunidades de pesquisa. Para isso foi realizado um Levantamento (survey) na literatura existente. Os resultados revelam que o estudo dessa temática ainda se encontra em estágio inicial, sendo diversas as possibilidades de pesquisas futuras.

Palavras-chave: tecnologias de informação móveis; tecnologias de informação sem fio; computação ubíqua; pesquisa em sistemas de informação; negócios móveis.

\begin{abstract}
Mobile, Wireless and Ubiquitous Information Technologies are main issues been discussed in the Information System area nowadays, both in the academic and business realms. The emerging applications of these technologies have brought a lot of questions about their design, ways of adoption, adaptation and consequences of use. However, due to the fact that they are very recent technologies, there is a conceptual confusion about terminologies such as: Mobile Technologies, Wireless Technologies and Ubiquitous Technologies, Mobile Commerce and Mobile Business. These terminologies are widely used in the business market and in academic research without a clear definition of their meaning. Considering this issue, this article attempts to map the State-of-Art of research about Mobile, Wireless and Ubiquitous Information Technologies, as well as it identifies some gaps of knowledge, indicating some research opportunities. To reach that goal, the article presents a Survey over the current literature. The results reveal that the research about Mobile, Wireless and Ubiquitous Technologies is in a very initial stage, with a lot of possibilities for future research.
\end{abstract}

Key words: mobile information technology; wireless information technology; ubiquitous computing; information systems research; mobile business. 


\section{INTRODUÇÃO}

As tecnologias Móveis, Sem Fio e Ubíquas estão entre os assuntos mais discutidos na área de Sistemas de Informação atualmente. Com o crescimento da telefonia móvel, banda larga e redes sem fio, a mobilidade e a computação em múltiplas plataformas e aparelhos tornam-se cada vez mais factíveis (Kalakota \& Robinson, 2002; Weiser, 1991, 1993; Watson, Pitt, Berthon, \& Zinkhan, 2002). A indústria de TI tem realizado intensa divulgação dessas tecnologias, argumentando que elas viabilizam os assim chamados "Negócios Móveis" (m-business) (Fenn \& Linden, 2001; Kalakota \& Robinson, 2002).

Apesar de certas restrições quanto a custo, disponibilidade, padrões universais e segurança, as Tecnologias de Informação Móveis e Sem Fio se propagam mundialmente e, da mesma forma, no mercado brasileiro. No Brasil, o número de telefones celulares (57 milhões) já ultrapassou o número de telefones fixos. (IDGNow/PCWorld, 2004). Várias empresas já estão instalando serviços de acesso à Internet via link sem fio em diversos ambientes, como aeroportos, cafés, hotéis, especialmente nas capitais do País (Fusco, 2003; IDGNow, 2003a; ComputerWorld, 2003). Além disso, laptops e PDAs (Personal Digital Assistants - Assistentes Digitais Pessoais), produzidos recentemente, já são adequados ao uso de redes sem fio (Idgnow/PCNews, 2003; Gartner Group como citado em IDGNow, 2003b). Telefones inteligentes (telefone celular e PDA com acesso à Internet em um único aparelho) começam a ser comercializados no País (IDGNow/PCWorld, 2003). Os telefones celulares ficam mais sofisticados, passando para as chamadas 2,5 e $3^{\text {a }}$ gerações. A RFID (Radio Frequency Identification - Identificação por Rádio Freqüência) começa a ser utilizada, especialmente no varejo (IDGNow/WorldTelecom, 2003). As redes sem fio como as WLAN (Wireless Local Area Network - Redes Locais Sem Fio) e acesso a dados, via aparelhos sem fio, estão se tornando comuns em algumas empresas, especialmente para apoiar atividades comerciais e de atendimento aos consumidores em campo.

A crescente aplicação dessas tecnologias faz emergir uma série de questões relativas à sua criação, escolha, adaptação e conseqüências de utilização. Contudo, por serem recentes, há certa confusão conceitual em torno de termos como: Tecnologias Móveis (Mobile), Tecnologias Sem Fio (wireless), Tecnologias Ubíquas, Comércio Móvel (m-commerce) e Negócios Móveis (m-business). Como será visto na sequiência, esses termos são utilizados em profusão, tanto no mercado quanto na pesquisa acadêmica, muitas vezes sem definição clara do seu significado. Acreditase que o primeiro passo para que se possa avançar no conhecimento desse tema é 
buscar uma definição mais precisa de termos. Dado o potencial de utilização dessas tecnologias, é importante também mapear que tipos de estudos estão sendo desenvolvidos a respeito, assim como as lacunas de pesquisa ainda existentes.

Diante desse contexto, o objetivo deste artigo é realizar um mapeamento do estado-da-arte da pesquisa sobre as Tecnologias de Informação Móveis, Sem Fio e Ubíquas, identificar as definições dos principais conceitos relacionados a essas tecnologias, bem como as oportunidades de pesquisa sobre essa temática. Para atingir esse objetivo, foi realizado um Levantamento (survey) na literatura existente sobre essas tecnologias.

Assim, na próxima seção é apresentado o método de pesquisa; na sequiência, a análise dos dados, procurando-se chegar às definições dos termos citados na literatura e à identificação dos principais temas abordados, bem como os métodos de pesquisa utilizados. Na seção seguinte, faz-se um apanhado da produção científica brasileira a respeito do tema de estudo. A partir dessas análises, são apresentadas as conclusões do estudo e a indicação de oportunidades de pesquisa futura. A sexta seção lista todos os artigos pesquisados via Levantamento e a seção final indica a produção brasileira analisada e outras referências bibliográficas utilizadas.

\section{Método de Pesquisa}

Foi realizado um Levantamento dos dados secundários (Fink, 1995) da literatura sobre as Tecnologias de Informação Móveis, Sem Fio e Ubíquas, selecionandose artigos disponíveis em bases de dados de pesquisa acadêmica na área de Gestão e Sistemas de Informação. As bases consultadas foram: ABI/Inform Global, EBSCO, Science Direct, Scirrus, e também o site da Association for Information Systems (AIS) - (http://www.aisnet.org). Foi selecionado um conjunto de 53 artigos, com o uso das seguintes palavras-chaves: mobile commerce; m-commerce; mobile business; m-business; ubiquitous computing; mobile computing; wireless e management e organization. Estes dois últimos termos foram utilizados, tendo em vista rastrear artigos que se relacionassem com temas organizacionais e de gestão, descartando-se artigos puramente técnicos. Foram selecionados artigos de periódicos e de dois congressos, todos possuindo comitê de avaliação; foram considerados somente artigos completos; não foram incluídos artigos que tratassem de setores específicos ou culturas nacionais, tendo em vista acessar estudos de maior abrangência.

Os artigos provêm dos seguintes periódicos: 7 artigos da Communications of The ACM; 3 artigos da Telematics and Informatics; 2 artigos de cada um dos 
seguintes periódicos: The Journal of Information Technology Theory and Application (JITTA); Applied Ergonomics; Journal of The Academy of Marketing Science; Journal of Computer Information System; Telecommunications Policy; Journal of Computer Assisted Learning. Um artigo de cada um dos seguintes periódicos: Computer Communications; Computer Networks; Electronic Commerce Research and Applications; Info Systems; Information and Management; Information Systems Management; Information Systems Research; Journal of Business Research; Journal of Business Strategies; Journal of Computer Assisted Learning; Journal of Electronic Commerce; Qualitative Marketing Research; Scientific American; Technological Forecasting and Social Change; Technovation; The Information Management Journal. Foram considerados artigos de duas conferências promovidas pela AIS: 5 artigos do ICIS (International Conference of Information Systems) e 10 artigos do AMCIS (Americas Conference of Information Systems). Todos os artigos foram publicados entre 1991 e 2004, sendo a maioria deles (49\% - 26 artigos) datados de 2002.

Primeiramente foi elaborada uma grade de coleta de dados, composta pelas seguintes variáveis: Referência Completa do artigo; Nome do Periódico/ Conferência; Ano de publicação; Conceito Central Abordado; Tema; Tecnologia Estudada; Método; Principais Resultados. Cada artigo foi lido, analisado, e os seus dados foram registrados em um software próprio para a análise de dados textuais. Foram criadas categorias de "respostas" para cada elemento levantado, para a posterior tabulação dos dados, chegando-se ao conteúdo apresentado nos quadros 1 a 4 da próxima seção (Análise dos Dados).

Cabe destacar que o Levantamento realizado não teve a pretensão de ser exaustivo, mas dada a qualidade e variedade das fontes acessadas, acredita-se que a amostra utilizada sinaliza os principais aspectos pesquisados em relação à temática tratada.

\section{AnÁlise dos Dados}

\section{Móvel, Sem Fio, Ubíqua, Pervasiva, Embutida, Nômade - Que Tecnologia é Essa?}

O primeiro aspecto identificado a partir do Levantamento na literatura é que diversos são os termos utilizados para se referir a dispositivos, aplicações, sistemas de informações ou mesmo transações de negócios com a utilização de Tecnologias de Informação Móveis, Sem Fio e Ubíquas. Exemplos: [tecnologias, sistemas ou 
aplicações] «móveis »; «sem fio »; «ubíquas »; « nômades ». Contudo, verificou-se que $41 \%$ dos artigos (22) não apresentam uma definição precisa do termo que está sendo utilizado. Cabe discutir a definição dos principais termos mencionados.

Tecnologias de Informação Móveis (mobile) - Mobilidade relaciona-se com portabilidade, isto é, a capacidade de se levar, para qualquer lugar, um dispositivo de Tecnologia de Informação (baseado em Kalakota \& Robinson, 2002). Logo, um laptop ou um PDA comum (sem capacidade de acesso a redes sem fio) são tecnologias móveis. Weilenmann (2003) vai além e diz que uma tecnologia móvel é aquela que é criada para ser usada enquanto se está em movimento (por exemplo, um walkman). No entanto ela ressalta que uma tecnologia móvel também é assim designada por possuir portabilidade.

Entretanto cabe considerar que, muitas vezes, quando se utiliza o termo mobile os autores estão se referindo ao uso de dispositivos de TI Móveis e Sem Fio (veja a definição na seqüência), isto é, aparelhos como, por exemplo, telefones celulares ou PDAs que podem ser conectados a uma rede e especialmente à Internet, via acesso sem fio. Ainda há casos em que o termo Móvel (mobile) e Sem Fio (wireless) são usados como sinônimos (veja como exemplo Tarasewich, Nickerson, \& Warketing, 2001).

Tecnologias de Informação Sem Fio (wireless) - São Tecnologias de Informação que envolvem o uso de dispositivos conectados a uma rede ou a outro aparelho por links de comunicação sem fio, como, por exemplo, as redes de telefonia celular ou a transmissão de dados via satélite, além das seguintes tecnologias: Infravermelho (infrared - IR), Bluetooth, Wireless LAN (Rede Local sem fio) (a respeito dessas tecnologias, veja Bobak, 2001) e Wi-Max (Telecomweb, 2004).

Em geral tende-se a associar o termo «sem fio » com mobilidade, mas isso nem sempre é verdadeiro. Por exemplo: pode-se ter um computador de mesa ligado a uma rede sem fio, mas nem por isso ele é um dispositivo móvel; o acesso à Internet em um "Hot Spot", envolve o uso de tecnologia sem fio, mas com mobilidade restrita àquele local; a troca de cartões de visita pela conexão via infravermelho entre dois PDAs também implica o uso de tecnologia sem fio, mas de mobilidade ainda mais restrita. Na verdade, a idéia de « wireless » é tão antiga quanto qualquer transmissão por rádio, porém agora se estende rapidamente a usos computacionais (Mannings \& Cosier, 2001).

Tecnologias de Informação Ubíquas (ubiquitous) - O termo ubíquo tem como uma de suas principais origens a definição apresentada no artigo seminal de Weiser (1991). Segundo o autor, estamos caminhando para a era da tecnologia calma (calm technology), quando a computação passa a ser subjacente às nossas 
vidas. Os computadores passam a ser tão naturais, tão sob medida e tão embutidos em todos os locais e nos mais diferentes objetos; eles tendem a se tornar praticamente invisíveis, isto é, nós os utilizaremos quase sem pensar, tal qual utilizamos a energia elétrica atualmente. Logo, a Computação Ubíqua envolve, em termos tecnológicos, a existência de diversos computadores interconectados por redes sem fios em cada ambiente, protocolos de comunicação que permitam o trânsito entre diferentes dispositivos e entre redes que se espalham pelos prédios, ruas, carros, em todos os locais. Isso demanda computadores de diferentes tamanhos, assim como sensores que os tornem conscientes de cada usuário e de cada ambiente.

Weiser $(1991,1996)$ estabelece uma diferenciação entre a Computação Ubíqua e os dispositivos móveis, uma vez que, diferentemente de PDAs, laptops ou telefones celulares, ela é invisível e onipresente, não havendo a necessidade de se carregar dispositivos específicos, já que a informação poderá ser acessada de qualquer lugar e em qualquer tempo, em diversos dispositivos de uso diário.

As tecnologias de redes sem fio, dispositivos de localização automática, sensores e dispositivos de RFID (Strassner \& Schoch, 2002), já existentes atualmente, são elementos chaves para a Computação Ubíqua. No entanto a Computação Ubíqua, tal qual definida originalmente por Weiser, ainda não é uma realidade, muito embora avanços tecnológicos estejam contribuindo para a sua difusão.

Cabe destacar que, em geral, os termos computação pervasiva (pervasive computing), ou embutida (embbeded) referem-se de certa forma ao conceito de computação ubíqua, muito embora o termo embutido se refira a apenas uma de suas características. De acordo com Abowd e Mynatt (como citado em Baber \& Baumann, 2002), aplicações de Computação Ubíqua compartilham três objetivos básicos: Interação natural com as pessoas, com objetos do dia-a-dia servindo de interfaces para ambientes computacionais; Tecnologias inteligentes, sensíveis a diferentes contextos e atividades humanas, capazes de reagir a elas e Comunicação, tanto pessoa-objetos quanto objetos-a-objetos.

Tecnologias de Informação Nômades (nomadic) - Lyytinen e Yoo (2002) propõem o termo Computação Nômade (Nomadic Computing). Eles asseveram que um ambiente de informação nômade é "uma confluência heterogênea de elementos tecnológicos, sociais e organizacionais interconectados, que possibilitam a mobilidade física e social de serviços de comunicação e computação entre atores, tanto dentro quanto fora das fronteiras organizacionais" (p. 377). Três características são chaves para a computação nômade: mobilidade, serviços/infra-estrutura em larga escala e convergência. Ela é focada na idéia de Tecnologias de Informação Móveis e Sem fio, mas chama a atenção para outros elementos relacionados a tecnologias que apóiam trabalhadores nômades, 
como questões relacionadas à interação homem-computador, psicologia, sociologia, ergonomia etc. Nessa visão ampliada sobre as tecnologias móveis, Kakihara e Sorensen (2002) e Sorensen (2003) argumentam que a mobilidade não possui somente uma dimensão espacial, mas também temporal e contextual

As Tecnologias de Informação Móveis e Sem fio têm possibilitado os chamados Comércio Móvel e Negócios Móveis. Outro conceito localizado foi o de Comércio Ubíquo. Cabe igualmente uma definição desses termos.

Comércio Móvel - Uma das definições mais completas de Comércio Móvel foi encontrada em Balasubramanian, Peterson e Javenpaa (2002). Os autores conceituam o Comércio Móvel (Mobile Commerce ou M-commerce) como qualquer fenômeno que possua as seguintes características: (1) envolve comunicação, de uma ou duas vias, entre dois ou mais humanos, entre humano(s) e um ou mais objeto inanimado, ou entre dois ou mais objetos inanimados (entre aparelhos); (2) para pelo menos uma das partes, a sua capacidade de comunicação não está vinculada a estar em determinado local, em ponto específico no tempo; (3) essa capacidade de se comunicar deve ser sustentável pelo menos por uma das partes durante uma movimentação substancial de um lugar para outro; (4) os sinais de comunicação entre as partes devem ser preferencialmente carregados por ondas eletromagnéticas; (5) se a comunicação se der entre humanos, pelo menos um deles procura beneficiar-se economicamente dessa comunicação, a curto ou longo prazo. Se a comunicação se der entre objetos, ela deve buscar criar benefícios econômicos para um humano ou empresa.

Os autores ressaltam que os objetivos do Comércio Móvel (que utiliza Tecnologias Móveis e Sem fio) são, em última instância, de natureza econômica. Nesse caso, os autores tomam como equivalente o termo Comércio Móvel ( $M$ commerce) e o termo negócios móveis (M-business). Cabe esclarecer que Comércio Móvel não é sinônimo de operações de Comércio Eletrônico via dispositivos Móveis e Sem Fio de acesso à Internet, pois ele é mais abrangente. Para uma análise mais completa das diferenças entre Comércio Eletrônico e Comércio Móvel, veja Zhang e Yuan (2002).

Comércio Ubíquo (U-Commerce) - Watson et al. (2002, p. 332) cunharam o termo U-Commerce, ou Comércio Ubíquo, definido como "o uso de redes ubíquas para apoiar comunicações personalizadas e ininterruptas e transações entre uma firma e seus vários stakeholders para oferecer um nível de valor além do comércio tradicional". Os autores oferecem um framework conceitual para a análise das possibilidades de utilização de aplicações de Comércio Ubíquo. Porém é importante observar que Watson et al. (2002) não utilizam a definição original de Computação Ubíqua proposta por Weiser (1991). 
Negócios Móveis - muito embora esse termo não tenha sido definido nos artigos levantados, encontram-se definições nos livros de Kalakota e Robinson (2002) e Paavilainen (2001). Este autor define Negócios Móveis como "a troca de bens, serviços e informação, utilizando-se tecnologias móveis" (p. 01). Já para Kalakota e Robinson (2002, p. 20), o termo engloba tanto as operações de Comércio Móvel (linha de frente) quanto "a infra-estrutura de aplicação necessária para manter relações de negócios e vender informações, serviços e mercadorias por meio dos aparelhos móveis". Logo, de acordo com esses autores, pode-se concluir que o conceito de Negócios Móveis engloba as operações de Comércio Móvel e todos os demais processos (operacionais e de gestão) que as tornam possíveis.

\section{Que Está Sendo Discutido ou Pesquisado Sobre Tecnologias de Informação Móveis, Sem Fio e Ubíquas?}

Quanto ao tipo de tecnologia alvo de estudos, o Quadro 1 revela que a maior parte dos artigos 54,7\% (29) discute diversas tecnologias Móveis, Sem Fio e Ubíquas (conforme a denominação usada em cada trabalho), isto é, a tecnologia é tratada de forma genérica, mencionando-se diversos tipos de aplicações. Cruzando-se variáveis, verifica-se que todos esses 29 artigos correspondem a ensaios ou discussões teóricas.

\section{Quadro 1: Tipos de Tecnologias Discutidas/Pesquisadas}

\begin{tabular}{|l|r|r|}
\hline \multicolumn{1}{|c|}{ Tecnologia 2 } & \multicolumn{1}{c|}{\begin{tabular}{l|l|} 
ct. \\
cit.
\end{tabular}} & Freq. \\
\hline Diversas tecnologias móveis/sem fio & 29 & $54,7 \%$ \\
\hline Telefones celulares c/ acesso Internet & 8 & $15,1 \%$ \\
\hline PDA & 3 & $5,7 \%$ \\
\hline Sistemas/Aplicativos empresariais móveis & 3 & $5,7 \%$ \\
\hline Telefones celulares & 2 & $3,8 \%$ \\
\hline Carro com dispositivos móveis (celular, notebook) & 1 & $1,9 \%$ \\
\hline Protocolo WAP & 1 & $1,9 \%$ \\
\hline Tecnologias "vestíveis" & 1 & $1,9 \%$ \\
\hline Notebooks com IR & 1 & $1,9 \%$ \\
\hline Telefones celulares com acesso à Internet e PDAs & 1 & $1,9 \%$ \\
\hline Telefones celulares com acesso à Internet, PDAs e redes sem fio & 1 & $1,9 \%$ \\
\hline Telefones celulares e BIPs & 1 & $1,9 \%$ \\
\hline Telefones celulares e PDAs & 1 & $1,9 \%$ \\
\hline TOTAL OBS. & $\mathbf{5 3}$ & $\mathbf{1 0 0 \%}$ \\
\hline
\end{tabular}


Em relação a tecnologias específicas, a mais abordada (8 artigos) são os telefones celulares com acesso à Internet, sendo 4 desses artigos referentes a pesquisas empíricas. Os PDAs são estudados por 3 pesquisas empíricas e os sistemas/aplicativos empresariais móveis são alvo de 2 pesquisas empíricas e um ensaio teórico. Quatro pesquisas empíricas estudaram mais de uma tecnologia de forma concominante.

Os temas centrais pesquisados são listados no Quadro 2, identificando-se os respectivos artigos de acordo com o número com que eles são listados na seção Conclusões. Cabe ressaltar que as categorias não são excludentes (alguns artigos abordam mais de um tema).

\section{Quadro 2: Principais Temas Discutidos/Pesquisados}

\begin{tabular}{|l|l|r|}
\hline \multicolumn{1}{|c|}{ TEMA } & \multicolumn{1}{c|}{ ARTIGOS } & QT \\
\hline Desafios Tecnológicos em relação à Computação Ubíqua & $4 ; 6 ; 13 ; 21 ; 30 ; 37 ; 45 ; 48 ; 50$ & 9 \\
\hline Efeitos do uso de tecnologias Móveis/Sem fio sobre trabalhadores & $9 ; 11 ; 14 ; 29 ; 33 ; 39 ; 52$ & 7 \\
\hline Tendências/questões genéricas relacionadas a Comércio Móvel/sem fio & $15 ; 16 ; 20 ; 46 ; 47$ & 5 \\
\hline Fatores Críticos para a adoção de TI Móveis & $27 ; 44 ; 1 ; 24 ; 43$ & 5 \\
\hline Aplicativos/Sistemas empresariais Móveis & $1 ; 9 ; 35 ; 39$ & 4 \\
\hline Análise da evolução de TI Móveis & $2 ; 8 ; 16 ; 32$ & 4 \\
\hline Análise de aspectos sociais relacionados a tecnologias ubíquas & $4 ; 13 ; 22 ; 26$ & 4 \\
\hline Aplicação de TI Móveis/Sem Fio para aprendizagem & $40 ; 33 ; 52$ & 3 \\
\hline Estudo da cadeia de valor do Comércio Móvel & $7 ; 36 ; 41$ & 3 \\
\hline Definição/discussão e aplicações do Comércio Ubíquo (U-commerce) & $28 ; 29 ; 49$ & 3 \\
\hline Possibilidades de criação de valor no Comércio Móvel & $3 ; 12$ & 2 \\
\hline Análise dos efeitos de TI Ubíqua na interação empresa-consumidores & $19 ; 50$ & 2 \\
\hline Avaliação de possíveis aplicações e resultados do uso de TI Móveis & $5 ; 11$ & 2 \\
\hline Questões socioeconômicas relacionadas ao Comércio Móvel & 8 & 1 \\
\hline Avaliação da Qualidade da Informação para acesso via Internet Móvel & 10 & 1 \\
\hline Gerenciamento da informação em dispositivos móveis/sem fio & 17 & 1 \\
\hline Uso do carro como escritório móvel & 18 & 1 \\
\hline Uso de TI móveis/sem fio por Pequenas e Médias Empresas & 23 & 1 \\
\hline Aplicações de TI Móveis de acordo com diferentes setores & 25 & 1 \\
\hline Conseq. sociais/organizacionais do uso tec. De comunicação sem fio & 31 & 1 \\
\hline Proposição do termo "Computação Nômade" (Nomadic Computing) & 34 & 1 \\
\hline Metodologias de desenvolvimento de sistemas p/ acesso móvel/sem fio & 35 & 1 \\
\hline Serviços de dados sem fio & 38 & 1 \\
\hline Importância da análise contextualista no estudo de TI Ubíquas & 42 & 1 \\
\hline Modelos de negócios de Comércio Móvel & 47 & 1 \\
\hline Definição/ Discussão do termo "Computação Ubíqua" (artigo seminal) & 51 & 1 \\
\hline Diferenças entre Comércio Eletrônico e Comércio Móvel & 53 & \\
\hline & &
\end{tabular}

Pode-se verificar que o escopo de temas pesquisados é relativamente amplo. Para facilitar a análise, na seqüência são analisados blocos comuns entre esses temas. 
Um dos temas mais presentes nos artigos (9) são os desafios tecnológicos em relação à Computação Ubíqua. Dentro desse escopo, podem-se verificar como focos a padronização de padrões de comunicação entre redes sem fio, segurança, design de aplicações, infra-estrutura, ergonomia, entre outros. Quatro artigos focam os aspectos sociais relacionados à Computação Ubíqua, pelo seu amplo espectro de possibilidades de sua aplicação.

O Comércio Móvel é foco de vários artigos. Alguns deles (5) analisam tendências e questões genéricas relacionadas ao Comércio Móvel (basicamente ensaios teóricos); 3 artigos focam a cadeia de valor do Comércio Móvel. Outros temas são: definição e aplicações de Comércio Ubíquo (3 artigos); possibilidades de criação de valor no Comércio Móvel (2 artigos); modelos de Negócios Móveis (1 artigo); diferenças entre Comércio Eletrônico e Comércio Móvel (1) questões sócio-econômicas relativas ao Comércio Móvel (1).

Outro tema central (presente em 7 artigos) são os efeitos do uso de Tecnologias Móveis e Tecnologias Sem Fio sobre trabalhadores (trabalhadores do conhecimento, executivos) e também sobre o processo de aprendizagem ( 3 artigos). $\mathrm{O}$ foco desses artigos em geral é como essas tecnologias podem contribuir para a eficiência e eficácia no trabalho.

Os fatores críticos para a adoção de tecnologias móveis são alvo de estudo de 5 artigos. Dois desses artigos baseiam-se no modelo TAM - Technology Acceptance Model (Davis, 1989); outros de alguma forma consideram variáveis desse modelo para a avaliação da aceitação de tecnologias Móveis/ Sem fio.

As possibilidades de aplicação e resultados gerais de uso das tecnologias Móveis/Sem fio também são exploradas nos artigos (2 focam esse tema diretamente); 1 artigo analisa o uso do carro como escritório móvel; 1 foca o uso de TI Móvel/Sem Fio por pequenas empresas; 1 discute a aplicação dessas tecnologias de acordo com diferentes setores empresariais. Os aplicativos e sistemas de informação Móveis e Sem Fio também são abordados pelos artigos (4), assim como os serviços de dados sem fio (1 artigo). Um artigo foca as metodologias de desenvolvimento de sistemas para acesso Móvel/Sem fio e um deles discute o gerenciamento da informação em dispositivos Móveis/ Sem Fio. Um artigo avalia especificamente a qualidade da Informação acessada via Internet Móvel.

Quanto aos métodos de pesquisa utilizados nos artigos levantados, como foi já foi mencionado, 35 dos 53 artigos (66\%) não apresentam pesquisa empírica. Entre os artigos que apresentam pesquisa empírica, a maioria utiliza o método 
de pesquisa survey (7 artigos); 3 artigos utilizam Estudos de Caso; 2 apresentam Experimentos. Alguns artigos utilizam Entrevistas (1) e Focus Group (1); 1 deles indica o uso de pesquisa-ação e dois deles baseiam-se na análise de dados secundários, um pela análise de séries históricas e outro pela análise de um conjunto de dados setoriais (veja Quadro 3).

\section{Quadro 3: Tipos de Métodos de Pesquisa Utilizados}

\begin{tabular}{|l|r|r|}
\hline \multicolumn{1}{|c|}{ Método_2 } & Qt. cit. & Freq. \\
\hline Sem pesquisa empírica & 35 & $66,0 \%$ \\
\hline Survey & 7 & $13,2 \%$ \\
\hline Estudo de caso & 3 & $5,7 \%$ \\
\hline Experimento & 2 & $3,8 \%$ \\
\hline Focus Group & 1 & $1,9 \%$ \\
\hline Entrevistas & 1 & $1,9 \%$ \\
\hline Pesquisa-ação & 1 & $1,9 \%$ \\
\hline Etnografia & 1 & $1,9 \%$ \\
\hline Análise de séries históricas & 1 & $1,9 \%$ \\
\hline Análise de dados setoriais & 1 & $1,9 \%$ \\
\hline TOTAL OBS. & $\mathbf{5 3}$ & $\mathbf{1 0 0 \%}$ \\
\hline
\end{tabular}

Quanto aos principais resultados dos artigos (veja Quadro 4), verificou-se que a maioria deles $(54,7 \%)$ resulta em uma análise e/ou proposições genéricas sobre as tecnologias, aplicações e respectivos elementos abordados. Nove dos 53 artigos fazem proposições ou recomendações com base em pesquisa empírica; 3 propõem um Framework Analítico (um deles com base em pesquisa empírica e dois sem pesquisa empírica); 2 propõem um Framework Conceitual, sem o uso de pesquisa empírica. Três artigos propõem modelos teóricos, dois com dados empíricos e um sem dados empíricos. Dois artigos adaptam e testam um modelo já existente - o modelo TAM (Davis, 1989) - e outros dois artigos propõem modelos a serem validados em pesquisa futura. 


\section{Quadro 4: Resultados dos Artigos Pesquisados}

\begin{tabular}{|l|r|r|}
\hline \multicolumn{1}{|c|}{ Resultados2 } & Qt. cit. & Freq. \\
\hline Análise/proposições genéricas s/pesquisa empírica & 29 & $54,7 \%$ \\
\hline Proposições/recomendações c/pesquisa empírica & 9 & $17,0 \%$ \\
\hline Framework Analítico & 3 & $5,7 \%$ \\
\hline Modelo teórico & 3 & $5,7 \%$ \\
\hline Framework conceitual & 2 & $3,8 \%$ \\
\hline Adaptação/aplicação modelo já existente & 2 & $3,8 \%$ \\
\hline Proposição de um modelo a ser pesquisado & 2 & $3,8 \%$ \\
\hline Taxinomia para class. aplicações móveis & 1 & $1,9 \%$ \\
\hline Hipóteses a serem testadas em pesquisa futura & 1 & $1,9 \%$ \\
\hline Definição conceitual (seminal) & 1 & $1,9 \%$ \\
\hline TOTAL OBS. & $\mathbf{5 3}$ & $\mathbf{1 0 0 \%}$ \\
\hline
\end{tabular}

\section{Como Está a Produção Científica Brasileira a Respeito das Tecnologias Móveis, Sem Fio ou Ubíquas?}

Concluída a pesquisa nas bases de dados internacionais, nos voltamos para a produção científica brasileira a respeito das tecnologias Móveis, Sem Fio e Ubíquas. Para isso, foram pesquisados os principais veículos de publicação acadêmica conceituados em nível "A" pela Capes na área de Administração, uma vez que, como já foi mencionado, a pesquisa visa rastrear artigos que se relacionem com temas organizacionais e de gestão. Foram selecionados os seguintes periódicos: RAC (Revista de Administração Contemporânea), RAE (Revista de Administração da Fundação Getúlio Vargas), RAUSP (Revista de Administração da USP) e Organizações e Sociedade (O\&S). Como congressos acadêmicos foram selecionados o ENANPAD (Encontro Nacional dos Programas de Pós-Graduação em Administração) e o ENEGEP (Encontro Nacional de Engenharia da Produção). Foram utilizadas as mesmas palavraschaves aplicadas à pesquisa nas bases de dados internacionais, tanto em português como em inglês, e o período de publicação considerado foi o de 1997 a 2004.

Não foram localizadas publicações a respeito do tema pesquisado na RAC, RAE e O\&S. Na RAUSP foi localizado o artigo de Anefalos e Caixeta Filho (2000) que realiza uma análise da utilização de sistemas de rastreamento por 
satélite em empresas de transporte rodoviário de cargas, através de um levantamento realizado junto a 26 empresas na cidade de São Paulo.

Quanto aos congressos científicos selecionados, nos anais do ENEGEP (19972004) foi localizado o artigo de Alverga e Ramos (2004), que realiza uma revisão teórica de conceitos relativos a m-business, mobilidade, redes sem fio e Internet móvel. O ENANPAD foi o veículo onde foi encontrado o maior número de publicações a respeito do tema de pesquisa. Livera e Moraes (2002) analisaram a competição na indústria de telefonia móvel celular e a introdução do Serviço Móvel Pessoal (SMP) por meio de uma pesquisa baseada em fontes secundárias. Kraft e Nique (2000) analisaram a experiência de uso do telefone celular por consumidores, utilizando a técnica ZMET (Zaltman Metaphor Elicitation Technique). Marques e João (2003) apresentam um levantamento junto a 73 executivos brasileiros a respeito de sua percepção, hábitos de uso e expectativas em relação a tecnologias Móveis (celular, PDA, Radiofreqüência etc.), utilizando o conceito de mobilidade e explorando suas características. Akabane e Nunes (2004) realizaram um estudo de caso sobre Comércio Móvel em empresa de logística, definindo e utilizando o conceito de $m$-commerce, e focando a aplicação de sistemas de comunicação via satélite.

Essa amostra indica que a produção científica brasileira a respeito de tecnologias Móveis, Sem Fio e Ubíquas até o momento pode ser considerada incipiente. Contudo cabe notar que os poucos estudos localizados se baseiam, em sua maioria, em pesquisas empíricas a respeito do tema. Isso indica a oportunidade e possibilidade de pesquisas futuras que ofereçam contribuições consistentes sobre o uso dessas tecnologias em nosso contexto.

\section{Conclusões e Identificação de Oportunidades para Pesquisas Futuras}

A seguir cada um dos principais resultados encontrados é resgatado e, a partir deles, são indicadas oportunidades e recomendações para pesquisas futuras.

Carência de definições teóricas claras. As definições sobre cada um dos tipos de Tecnologias de Informação - Móveis, Sem Fio e Ubíquas - em geral ainda não são claras, já que boa parte dos artigos pesquisados se refere a essas tecnologias, utilizando termos que não são definidos formalmente. Da mesma forma, muitas vezes utilizam-se os termos Móvel (Mobile), Sem Fio (wireless) e Ubíquo (ubiquitous) como se eles fossem sinônimos, quando na verdade, como já foi discutido, cada um deles possui características distintas. Recomenda-se 
que pesquisas futuras sobre essas temáticas atentem para a definição precisa dos termos utilizados, porque, do contrário, dificilmente se conseguirá gerar um corpo teórico robusto a respeito dessas tecnologias.

Tecnologias distintas tratadas de forma genérica. A maioria dos estudos aborda as Tecnologias de Informação Móveis, Sem Fio ou Ubíquas de forma genérica, discutindo diversas aplicações, mas sem foco no estudo de tecnologias específicas.

Deve-se considerar que cada dispositivo, sistema ou aplicação poderá ter usos e efeitos distintos que precisam ser estudados em profundidade. Cada uma delas implica um processo de hospedagem (Ciborra, 2002) distinto, com interações de pessoas, propriedades institucionais e tecnologia (Orlikowski, 1992) distintas.

Escopo amplo de temas pesquisados. O escopo de temas abordados é relativamente amplo. Foram identificados alguns temas freqüientes como: os desafios tecnológicos em relação à Computação Ubíqua; as aplicações e possibilidades de uso do Comércio Móvel; os efeitos do uso de Tecnologias Móveis e Sem Fio sobre os trabalhadores, com foco nos efeitos sobre eficiência, eficácia e produtividade, entre vários outros.

A partir do Levantamento realizado, puderam ser identificadas algumas lacunas que constituem oportunidades para pesquisas futuras. Entre as principais estão as seguintes.

. Estudo de questões sociais e comportamentais relacionadas à utilização de Tecnologias de Informação Móveis, Sem Fio e Ubíquas. Uma vez que muitos dos estudos sobre os efeitos do seu uso sobre os trabalhadores focam somente aspectos "racionais" e econômicos (eficiência, eficácia, produtividade, etc.). Como exemplo disso, Grudin (2002) coloca que as implicações sociais da computação ubíqua são evidentes, mas raramente consideradas cuidadosamente.

- Alinhamento Estratégico do uso de Tecnologias de Informação Móveis, Sem Fio e Ubíquas. Qualquer forma de utilização dessas tecnologias deverá estar relacionada com a estratégia organizacional. Em função da estratégia, serão escolhidos os produtos, processos, formas estruturais e as competências organizacionais que poderão ser potenciadas a partir da utilização dessas tecnologias. O estudo desse tema pode apoiar as organizações que planejam adotá-las.

. Avaliação de resultados do uso de Tecnologias de Informação Móveis, Sem Fio e Ubíquas. Uma vez que essas tecnologias são relativamente recentes, ainda há certa divergência no mercado quanto à avaliação de retorno, tanto por critérios 
quantitativos quanto qualitativos. A elaboração de modelos ou metodologias para avaliação de resultados da adoção dessas tecnologias é bastante pertinente e ainda pouco desenvolvida.

- Mudanças estruturais decorrentes de adoção de Tecnologias de Informação Móveis, Sem Fio e Ubíquas. Por sua abrangência e possibilidades de aplicação, é preciso investigar o potencial dessas tecnologias para viabilizar mudanças estruturais nas organizações, como, por exemplo, mudanças quanto ao grau de autonomia de trabalhadores em campo, potenciação de redes de trabalho, "achatamento" da estrutura, entre outras.

- Redesenho de processos organizacionais com a utilização de Tecnologias de Informação Móveis, Sem Fio e Ubíquas. É preciso investigar o potencial dessas tecnologias para possibilitar o redesenho e melhoria de processos organizacionais (eficiência/eficácia), quais os tipos de processos mais propensos a isso e quais as formas de redesenho.

. Processos de Aprendizagem e de Gestão do Conhecimento com a utilização de Tecnologias de Informação Móveis, Sem Fio e Ubíquas. Essas tecnologias podem possibilitar diversas mudanças nas formas pelas quais as pessoas se comunicam, interagem e compartilham informações e conhecimento. É interessante explorar de que forma elas podem colaborar com a Gestão do Conhecimento nas organizações.

Carência de pesquisa empírica. Como foi visto, a maioria dos artigos pesquisados nas bases de dados internacionais não apresentam pesquisa empírica. Entre os que apresentam, a maior parte utiliza o método de pesquisa Survey. Dado o estágio inicial de desenvolvimento do corpo teórico a respeito das Tecnologias de Informação Móveis, Sem Fio e Ubíquas, acredita-se que é fundamental a realização de pesquisas empíricas e que estas façam uso não só de pesquisas quantitativas, mas também qualitativas. Métodos como o Estudo de Caso, por exemplo, são adequados, quando se visa ao conhecimento mais profundo de uma realidade para a construção de uma proposição teórica (Benbasat, Goldstein, \& Mead, 1987).

Resultados de pesquisa pouco consistentes. A maioria dos artigos pesquisados tem como resultado análises ou proposições genéricas. Poucos são os que propõem Frameworks, modelos ou proposições com base em dados empíricos. Esse dado indica uma oportunidade para pesquisas futuras que visem a resultados mais concretos.

Pode-se concluir que os estudos sobre as Tecnologias Móveis, Sem Fio e Ubíquas se encontram em estágio bastante inicial, com um corpo teórico ainda em formação 
(veja Reinhard (1998), que comenta o modelo de ciclo de produto proposto em Kahin e Wilson (1996), fornecendo interessante perspectiva de análise do ciclo de vida do estudo da tecnologia).

Atualmente, há todo um discurso que proclama essas tecnologias como condutoras de um novo boom tecnológico, que lembra a febre das empresas ponto com no final do século passado. No entanto, ainda há muito a ser discutido e estudado a respeito dessa promessa tecnológica, especialmente no contexto brasileiro, para que se possa avaliar com maior nitidez as perdas e ganhos da adoção dessas tecnologias. Acredita-se que essas tecnologias abrem amplo leque de aplicações organizacionais, e, conseqüentemente, de possibilidades e demandas de pesquisa.

\section{Artigo recebido em 05.11.2004. Aprovado em 19.02.2005.}

\section{Agradecimentos}

Este artigo foi revisado e editado durante a realização do estágio de doutorando no exterior de Saccol, Amarolinda, com o apoio da CAPES. A autora agradece a Fabio Saccol e a Licia M. Abe a ajuda no processo de revisão final do artigo.

\section{ReferênCIAS Bibliográficas}

Akabane, G. K., \&

Nunes, L. C. (2004).

Os benefícios do comércio móvel em logística: uma pesquisa exploratória na Cesari empresa multimodal de movimentação de materiais Ltda. Anais do Encontro Nacional dos Programas de Pós-Graduação em Administração, Curitiba, PR, Brasil, 28.

Alverga, P., \&

Ramos, A. (2004).

Evolução, aplicações, barreiras e tendências do m-business. Anais do Encontro Nacional de Engenharia de Produção, Florianópolis, SC, Brasil, 24.
Anefalos, L., \&

Caixeta Filho, J. (2000).

Análise da utilização de sistemas de rastreamento por satélite em empresas de transporte rodoviário de cargas. Revista de Administração, 35(4), 22-35.

Baber, C., \&

Baumann, K. (2002).

Embedded human computer interaction. Applied Ergonomics, 33(3), 273-287. 
Balasubramanian, S.,

Peterson, R. A., \&

Javenpaa, L. (2002).

Exploring the implications of $\mathrm{m}$-commerce for markets and marketing. Journal of the Academy of Marketing Science, 30(4), 348-361.

Benbasat, I.,

Goldstein, D.K., \&

Mead, M. (1987).

The case research strategy in studies of information systems. MIS Quarterly, 11(3), 369-386.

Bobak, A. (2001).

Eletronic business with mobile personal digital assistants (PDAs). Master Thesis in Computer Science, University of Zurich, Switzerland.

Ciborra, C. (2002).

The labyrinths of information: challenging the wisdom of systems ( $\mathrm{p}$. 195). New York: Oxford Press.

ComputerWorld (2003).

IG lança serviço de acesso WI-FI. Recuperado em 16 julho, 2003, de http://www.computerworld.com.br

Davis, F. D. (1989).

Perceived usefulness, perceived ease of use, and user acceptance of information technology. MIS Quarterly, 13(3), 319-340.

Fenn, J., \&

Linden, A. (2001).

Trends for 2002 to 2007: up the slope of enlightenment. Gartner Group Article Top View. Recuperado em 23 abril, 2003, de http://www.gartner.com/ DisplayDocument?doc_cd=103538
Fink, A. (1995).

The survey handbook: Vol. 1. The Survey Kit. Thousand Oaks: Sage.

Fusco, C. (2003).

Terra aposta no acesso sem fio. Recuperado em 7 outubro, 2003, de http://www.idgnow.com.br

Grudin, J. (2002).

Group dynamics and ubiquitous computing. Communications of the ACM, 45(12), 74-78.

IDGNow (2003a).

Brasil Telecom lança serviço Wi-Fi com 90 hotspots. Recuperado em 2 dezembro, 2003, de http://www. idgnow.com.br

IDGNow (2003b).

Gartner: $80 \%$ dos notebooks comerciais terão Wi-Fi em 2005, 2003. Recuperado em 1 outubro, 2003, de http://www.idgnow.com.br

IDGNow/PCNews (2003).

Novos Palms chegam com Wi-Fi e câmera digital. Recuperado em 23 abril, 2003, de http://www.idgnow.com.br

IDGNow/PCWorld (2003).

Handspring chega oficialmente ao Brasil. Recuperado em 07 maio, 2003, de http://www.idgnow.com.br

IDGNow/PCWorld (2004).

Brasil tem 57 milhões de celulares. Recuperado em 20 setembro, 2004, de http://www.idgnow.com.br

IDGNow/WorldTelecom (2003).

Varejo pode trocar código de barras por radiofreqüência. Recuperado em 12 junho, 2003, de http:// www.idgnow.com.br 
Kahin, B., \&

Wilson, E. (Eds.). (1996).

National information infrastructure initiatives: vision and policy design. Cambridge, Mass.: MIT Press.

Kakihara, M., \&

Sorensen, C. (2002).

Mobility: an extended perspective. Proceedings of the Hawaii International Conference on System Sciences (IEEE), Hilton Waikoloa Village, Hawaii, USA, 35.

Kalakota, R., \&

Robinson, M. (2002). M-business: tecnologia móvel e estratégia de negócios (p. 249). Porto Alegre: Bookman.

Kraft, S., \&

Nique, W. M. (2000).

Desvendando o consumidor através das metáforas: uma aplicação da Zaltman metaphor elicitation technique (ZMET). Anais do Encontro Nacional dos Programas de Pós-Graduação em Administração, Florianópolis, SC, Brasil, 24.

Livera, O., \&

Moraes, W. (2002).

Hipercompetição na indústria de telefonia móvel celular: novo cenário com a introdução do serviço móvel pessoal autoria. Anais do Encontro Nacional dos Programas de PósGraduação em Administração, Salvador, BA, Brasil, 26.

Lyytinen, K., \&

Yoo, Y. (2002).

Research commentary: the next wave of nomadic computing. Information Systems Research, 13(4), 377-388.

Mannings, R., \&

Cosier, G. (2001).

Wireless everything - unwiring the world. BT Technology Journal, 19(4), 65-76.

Marques, E., \&

João, B. N. (2003).

Mobilidade: uma investigação de uso por executivos brasileiros. Anais do Encontro Nacional dos Programas de Pós-Graduação em Administração, Atibaia, SP, Brasil, 27.

Orlikowski, W. (1992).

The duality of technology: rethinking the concept of technology in organisations. Organisation Science, 3(3), 398-427.

Paavilainen, J. (2001).

Mobile business strategies: understanding the technologies and opportunities. London: IT Press.

Reinhard, N. (1998).

Transformações do paradigma da infra-estrutura da informação. Revista de Sociologia e Política, 10(11), 237238.

Sorensen, C. (2003).

Research issues in mobile informatics: classical concerns, pragmatic issues and emerging discourses. Workshop on Ubiquitous Working Environment at Whitehead School of Management, Cleveland, Ohio, USA. Recuperado em 05 outubro, 2004, de http:// mobility.is.lse.ac.uk 
Strassner, M., \&

Schoch, T. (2002).

Today's impact of ubiquitous computing on business processes. Recuperado em 01 outubro, 2003, de http://www.vs.inf.ethz.ch/publ/ papers/Strassner-Schoch-ImpactUbicomp.pdf

Tarasewich, P.,

Nickerson, R., \&

Warkenting, M. (2001).

Wireless/mobile e-commerce: technologies, applications and issues. Anais do Americas Conference on Information Systems, Boston, MA, Estados Unidos, 7.

Telecomweb (2004).

WiMax é nova aposta das operadoras. Recuperado em 23 março, 2004, de http://www.telecomweb.com.br

Watson, R.,

Pitt, L.,

Berthon, P., \&

Zinkhan, G. (2002).

U-commerce: expanding the universe of marketing. Journal of the Academy of Marketing Science, 30(4), 329-343.

Weilenmann, A. (2003).

Doing mobility. Unpublished doctoral dissertation, University of Göteborg, Sweden.

Weiser, M. (1991).

The computer for the 21 st Century. Scientific American, 265, 94-104.

Weiser, M. (1993).

Hot topics in ubiquitous computing. Computer, 26(10), 71-72.

Weiser, M. (1996).

Ubiquitous computing. Recuperado em 21 junho, 2003, de http:// www.ubicomp.com/hypertext/weiser/ ubihome.html

Zhang, J., \&

Yuan, Y. (2002).

M-commerce versus internet-based ecommerce: the key differences. Proceedings of the Americas Conference on Information Systems, Dallas, Texas, USA, 8. 
APÊNDICE

\section{ReferênCIAs Bibliográficas dos 53 Artigos do LeVAntamento}

Abraham, D. (2001).

Mobile enterprise computing and computing and the diffusion of mobile enterprise business applications in organizations. Proceedings of the Americas Conference on Information Systems, Boston, Massachusetts, USA, 7 .

Allen, J. P. (2002).

Where enrollment meets structure: understanding PDA evolution through sociotechnological theory. Proceedings of the Americas Conference on Information Systems, Dallas, Texas, USA, 8.

Anckar, B., \&

D’Incau, D. (2002).

Value creation in mobile commerce: findings from a consumer survey. The Journal of Information Technology Theory and Application (JITTA), 4(1), 43-64.

Baber, C., \&

Baumann, K. (2002).

Embedded human computer interaction. Applied Ergonomics, 33(3), 273-287.

Balasubramanian, S.,

Peterson, R., \&

Javenpaa, S. (2002).

Exploring the implications of $\mathrm{m}$ commerce for markets and marketing. Journal of The Academy of Marketing Science, 30(4), 348-361.
Banavar, G., \&

Bernstein, A. (2002).

Software infrastructure and design challenges for ubiquitous computing applications. Communications of the ACM, 45(12), 92-96.

Barnes, S. J. (2003).

The mobile commerce value chain: analysis and future developments. International Journal of Information Management, 22(2), 91-108.

Beaubrun, R., \&

Pierre, S. (2001).

Technological developments and socio-economic issues of wireless mobile communication. Telematics and Informatics, 18(2-3), 143-158.

Beulen, E., \&

Streeng, R. (2002).

The impact of online mobile office applications on the effectiveness and efficiency of mobile workers' behavior: a field experiment in the IT services sector. Proceedings of the International Conference on Information Systems, Barcelona, Spain, 23.

Chae, M., \&

Kim, J. (2001).

Information quality for mobile internet services: a theoretical model with empirical validation. Proceedings of the International Conference on Information Systems, New Orleans, Louisiana, USA, 22. 
Chen, W. (2001).

Mobile communication technology and organizations: impacts service qualities, employee satisfaction and performance. Proceedings of the Americas Conference on Information Systems, Boston, Massachusetts, USA, 7.

Clarke III, I. (2001).

Emerging value propositions for $\mathrm{m}$ commerce. Journal of Business Strategies, 18(2), 133-148.

Crisler, K.,

Anneroth, M.,

Aftelak, A., \&

Pulil, P. (2003).

The human perspective of the wireless world. Computer Communications, 26(1), 11-18.

Davis, G. (2002).

Anytime/anyplace computing and the future of knowledge work. Communications of the ACM, 45(12), 67-73.

Deans, C. (2002).

Global trends and issues for mobile/ wireless commerce: the key differences. Proceedings of the Americas Conference on Information Systems, Dallas, Texas, USA, 8.

Dekleva, S. (2002).

M-business: economy driver or a mess. Proceedings of the Americas Conference on Information Systems, Dallas, Texas, USA, 8.

Elliot, R. (2002).

Wireless information management. The Information Management Journal, 36(5), 62-66.
Eost, C., \&

Flyte, M. G. (1998).

An investigation into the use of the car as a mobile office. Applied Ergonomics, 29(5), 383-388.

Fano, A., \&

Gershman, A. (2002).

The future of business services in the age of ubiquitous computing. Communications of the ACM, 45(12), 83-87.

Frolick, M. N., \&

Chen, L. D. (2004).

Assessing m-commerce opportunities. Information Systems Management, 21(2), 53-61.

Gerstheimer, O., \&

Lupp, C. (2004).

Needs versus technology - the challenge to design third-generation mobile applications. Journal of Business Research, 57(12), 1409-1415.

Grudin, J. (2002).

Group dynamics and ubiquitous computing. Communications of the ACM, 45(12), 74-78.

Harker, D., \&

Akkeren, J. (2002).

Exploring the needs of SMEs for mobile data technologies: the role of qualitative research techniques. Qualitative Marketing Research, 5(3), 199-209.

Hung, S. Y.,

Ku, C. Y., \&

Chang, C. M. (2003).

Critical factors of WAP services adoption: an empirical study. Eletronic Commerce Research and Applications, 2(1), 42-60. 
Jain, R. (2003).

Enterprise mobile services: framework and industry-specific analysis. Proceedings of the Americas Conference on Information Systems, Tamba, Florida, USA, 9.

Jessup, L., \&

Robey, D. (2002).

The relevance of social issues in ubiquitous computing environments. Communications of the ACM, 45(12), 88-91.

Jih, W. K., \&

Lee, S. (2003-2004).

An exploratory analysis of relationships between cellular phones uses' shopping motivators and lifestyle indicators. Journal of Computer Information Systems, 44(2), 65-73.

Junglas, I., \&

Watson, R. (2003a).

U-commerce: an experimental investigation of ubiquity and uniqueness. Proceedings of the International Conference on Information Systems, Seattle, WA, USA, 24.

Junglas, I., \&

Watson, R. (2003b).

U-commerce: a conceptual extension of e-commerce and m-commerce. Proceedings of the International Conference on Information Systems, Seattle, WA, USA, 24.

Juul, N. C., \&

Jorgensen, N. (2003).

The security role in WAP: an analysis of the network and business rationales underlying a failure. International Journal of Eletronic Commerce, 7(4), 73-92.

Katz, J.E. (1997).

Social and organizational consequences of wireless communications: a selective analysis of residential and business sectors in the United States. Telematics and Informatics, 14(3), 233-256.

Kumar, S., \&

Zahn, C. (2003).

Mobile communications: evolution and impact on business operations. Technovation, 23(6), 515-520.

Lundin, J., \&

Magnusson, M. (2003).

Collaborative learning in mobile work. Journal of Computer Assisted Learning, 19(3), 273-283.

Lyytnen, K., \&

Yoo, Y. (2002).

Research commentary: the next wave of nomadic computing. Information Systems Research, 13(4), 377-388.

Olla, P.,

Atkinson, C., \&

Gandceha, R. (2003).

Wireless systems development methodologies: an analysis of practice using actor-network theory. Journal of Computer Information System, 44(1), 102-111.

Olla, P., \&

Patel, N. (2002).

A value chain model for mobile data services providers. Telecommunications Policy, 26(9-10), 551-571. 
Pierre, S. (2001).

Mobile computing and ubiquitous networking: concepts, technologies and challenges. Telematics and Informatics, 18(2-3), 109-131.

Preez, G., \&

Pistorius, C. W. I. (2003).

Analyzing technological threats and opportunities in wireless data services. Technological Forecasting and Social Change, 70(1), 1-20.

Puuronen, S., \&

Savolainen, V. (1997).

Mobile information systems executive's view. Information Systems Journal, 7(1), 3-20.

Roschelle, J. (2003).

Keynote paper: unlocking the learning value of wireless mobile devices.

Journal of Computer Assisted Learning, 19(3), 260-272.

Sabat, H. K. (2002).

The evolving mobile wireless value chain and market structure. Telecommunications Policy, 26(9-10), 505-535.

Scheepers, R., \&

Scheepers, H. (2003).

Contexts of relevance in exploratory case studies in information systems: ubiquitous information technology implementation in organizations. Proceedings of the International Conference on Information Systems, Seattle, WA, USA, 24.
Sharma, S., \&

Deng, X. (2002).

An empirical investigation of factors affecting the acceptance of personal digital assistants by individuals. Proceedings of the Americas Conference on Information Systems, Dallas, Texas, USA, 8.

Shim, J. P.,

Bekkering, E., \&

Hall, L. (2002).

Empirical findings on perceived value of mobile commerce as a distribution channel. Proceedings of the Americas Conference on Information Systems, Dallas, Texas, USA, 8.

Siewiorek, D. (2002).

New frontiers of application design. Communications of the ACM, 45(12), 79-82.

Tarasewich, P.,

Nickerson, R., \&

Warkenting, M. (2001).

Wireless/mobile e-commerce: technologies, applications and issues. Proceedings of the Americas Conference on Information Systems, Boston, Massachusetts, USA, 7.

Tsalgatidou, A., \&

Pitoura, E. (2001).

Business models and transactions in mobile eletronic commerce: requirements and properties. Computer Networks, 37(2), 221-236.

Varshney, U. (2002).

Multicast over wireless networks. Communications of the ACM, 45(12), 31-37. 
Watson, R.,

Pitt, L.,

Berthon, P., \&

Zinkhan, G. (2002).

U-commerce: expanding the universe of marketing. Journal of The Academy of Marketing Science, 30(4), 329-343.

Weippl, E. (2001).

The transition from e-commerce to $\mathrm{m}$ commerce: why security should be the enabling technology. The Journal of Information Technology Theory and Application (JITTA), 3(4), 17-19.
Weiser, M. (1991).

The computer for the 21 st Century. Scientific American, 265, 94-104.

Weitz, R. R. (1997).

Lessons from notebook/mobile computing. Information and Management, 32(2), 103-111.

Zhang, J., \&

Yuan, Y. (2002).

M-commerce versus internet-based ecommerce: the key differences. Proceedings of the Americas Conference on Information Systems, Dallas, Texas, USA, 8. 